\title{
Magnetism in BEDT-TTF materials
}

\author{
R. T. Clay, ${ }^{a}, *$ S. Mazumdar ${ }^{b}$ \\ ${ }^{a}$ Department of Physics and Astronomy, ERC Center for Computational Sciences, Mississippi State University, \\ Mississippi State, MS, 39762, USA \\ ${ }^{\mathrm{b}}$ Department of Physics, University of Arizona, Tucson, AZ, 85721, USA
}

\begin{abstract}
Strong commensurate antiferromagnetism proximate to superconductivity is found in some members of the $\kappa$-(ET) family, while a spin gap (SG) is found in the $\theta$-(ET). Both $\kappa$ - and $\theta$-(ET) materials have frustrated triangular lattice structures. We show from calculations of spin-spin correlations within the effective half-filled band triangular lattice proposed for the $\kappa$-ET, as well as for the real lattice, that long range AFM order is not obtained as a consequence of this frustration. We argue that some other mechanism reduces the magnetic frustration in these systems. We show that the low temperature magnetic states in these materials can only be understood if the effects of the cooperative charge and bond ordering transitions occurring at higher temperatures in these systems are taken into account. In the $\kappa$-ET, this co-operative transition leads to unequal hole populations on the ET dimers that form the triangular lattice.
\end{abstract}

Keywords: Organic conductors based on radical cation and/or anion salts, organic superconductors

\section{Introduction}

The family of BEDT-TTF (ET) salts $\kappa-(\mathrm{ET})_{2} \mathrm{X}$ feature the highest known $\mathrm{T}_{c}$ of the organic superconductors. In $\kappa$-(ET), antiferromagnetism (AFM) is found adjacent to superconductivity (SC) as in the high- $\mathrm{T}_{c}$ cuprate superconductors, leading to speculation that the occurrence of AFM and SC must be related to each other $[1,2]$. Within existing theories of $\mathrm{AFM}$ and $\mathrm{SC}$ in the $\kappa-\mathrm{ET}_{2} \mathrm{X}$, these materials lie on both sides of a Mott-Hubbard metal-insulator transition, as described within a triangular lattice half-filled Hubbard Hamiltonian $[1,3]$. The schematic crystal structure of the $\kappa$ ET lattice is shown in Fig. 1(a), where pairs of ET molecules with nearly parallel molecular planes form closely spaced dimers, with nearest neighbor dimers having nearly orthogonal orientations, and an average of one half hole per ET molecule. Because the intradimer hopping integrals are much larger than the interdimer hopping integrals [4], the $\kappa$ lattice is an effective anisotropic triangu-

* Corresponding author: email rtc29@ra.msstate.edu
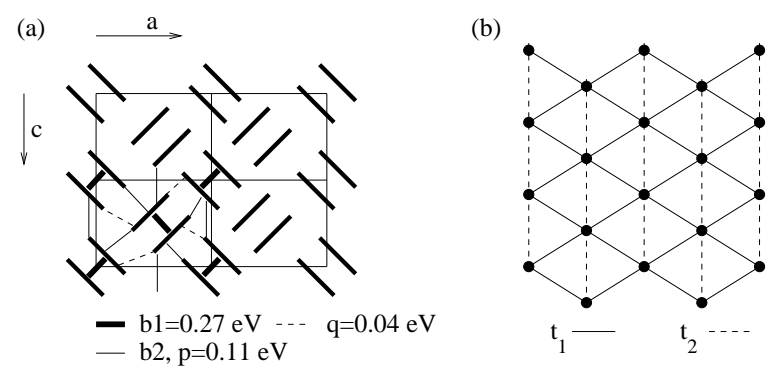

Fig. 1. (a) Schematic lattice structure of $\kappa$-(ET), including the intermolecular hopping integrals b1, b2 and q [4]. (b) Anisotropic triangular dimer lattice; each dimer of (a) constitutes one lattice point in this effective $\rho=1$ lattice. With the indicated values of $t_{b 2}, t_{p}$, and $t_{q}, t_{2} / t_{1}=0.73$ and $J_{2} / J_{1}=0.54$.

lar lattice of Fig. 1(b), each site within which is one dimer of Fig. 1(a). The ratio $t_{2} / t_{1}$ (note that here we denote the smaller frustrating bond as $t_{2}$ ) is $t_{2} / t_{1}=t_{b 2} /\left(t_{p}+t_{q}\right)$. Literature survey gives $t_{2} / t_{1}=0.5-0.7$ for $\mathrm{X}=\mathrm{Cu}\left[\mathrm{N}\left(\mathrm{CN}_{2}\right] \mathrm{Br}, 0.73\right.$ for $\mathrm{X}=\mathrm{Cu}\left[\mathrm{N}\left(\mathrm{CN}_{2}\right] \mathrm{Cl}, 1.0-1.10\right.$ for $\mathrm{X}=\mathrm{Cu}_{2}(\mathrm{CN})_{3}, 0.55$ - 0.6 for $\mathrm{X}=\mathrm{I}_{3}$ and $0.6-0.9$ for $\mathrm{X}=\mathrm{Cu}(\mathrm{SCN})_{2}$. The large $t_{2} / t_{1}$ imply that frustration plays a significant role in these systems. 
Despite the anticipated large frustration, two important experimental features must be noted: (a) when AFM order does occur, it is commensurate and (b) the (staggered) magnetic moment can be large. For example, the magnetic moment in $\kappa-(\mathrm{ET})_{2} \mathrm{Cu}\left[\mathrm{N}(\mathrm{CN})_{2}\right] \mathrm{Cl}$ is $0.45 \mu_{B} /$ dimer [5]. This value is comparable to the magnetization per site in the isotropic square lattice Heisenberg spin Hamiltonian. Considering the triangular dimer lattice structure of the $\kappa$-ET lattice, the experimentally observed magnetic moments are inexplicably large, and we argue below that they cannot be understood within existing theoretical models. Mean field (Hartree/Hartree-Fock) calculations are often cited as correctly reproducing the experimental magnitude of the AFM moment [3]. It is, however, well known that mean field theory greatly exaggerates broken symmetry in correlated systems: for example Hartree-Fock incorrectly predicts long-range $\mathrm{AFM}$ in one dimension (1D), and gives qualitatively incorrect predictions for charge order $(\mathrm{CO})$ in $1 \mathrm{D}$ as well as $2 \mathrm{D}[6,7]$.

The anisotropic triangular lattice has been studied by many authors, both within the Heisenberg spin Hamiltonian [8,9], and the half-filled Hubbard Hamiltonian $[3,10]$. Within the Heisenberg Hamiltonian the antiferromagnetic phase can correspond to either the three-sublattice spiral phase or the two-sublattice collinear phase [1]. The spiral phase is commensurate only at $\alpha=J_{2} / J_{1}=1$, where $J_{1} \sim t_{1}^{2} / U$ and $J_{2} \sim t_{2}^{2} / U$, respectively, and is precluded from experiment. The calculated magnetic moment in the collinear commensurate phase decreases rapidly as $\alpha$ increases from zero, and vanishes at $\alpha \sim 0.5$ [8]. Estimates of $\alpha$ in $\kappa$-(ET) range from 0.3 to 1.0. Only in the region of very small $\alpha$ are the calculated magnetic moments anywhere near the experimental values of $0.3-0.4$ $\mu_{B}$. A recent Hubbard model calculation [10] on the anisotropic triangular lattice (Fig.1(b)) using a path integral renormalization group approach for $2<U / t_{1}<10$ found a nonmagnetic insulating state over a broad region of $t_{2} / t_{1}$. For example, at $U / t_{1}=6, \mathrm{AFM}$ is only present for $t_{2} / t_{1}<0.5$ (corresponding to $\alpha<0.25$ ), smaller than the estimated $\alpha$ values for any $\kappa-(\mathrm{ET})_{2} \mathrm{X}[10]$.

From the above theoretical studies of the anisotropic triangular lattice it becomes clear that the effective half-filled model is not able to account for the large AFM moment observed in $\kappa$-(ET). In this paper we investigate theoretically the ground state of the anisotropic triangular lattice, both in the effective half-filled limit (Fig. 1(b)), as well as the quarter-filled lattice (Fig. 1(a)). Our results confirm the previous result [10] that the $\kappa$-(ET) system, considered from either the viewpoint of

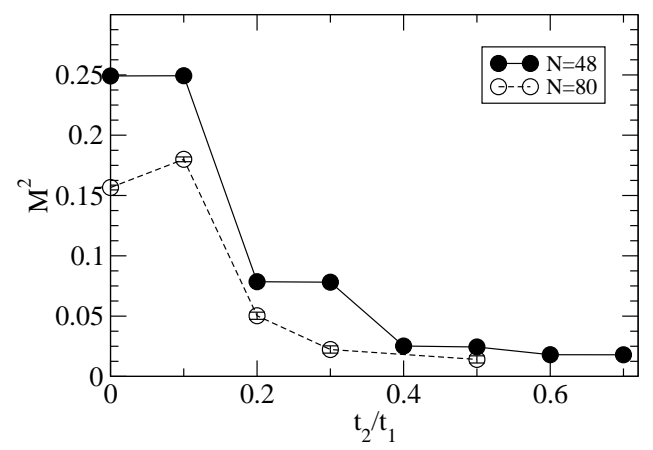

Fig. 2. Sublattice magnetization for the anisotropic triangular half-filled Hubbard model of Fig. 1(b) for 48 and 80 site lattices.

the effective half-filled band or the true quarterfilled model does not support an AFM state with large moments. We then ask the question as to whether the strong moment can be explained by the presence of an additional broken symmetry in the lattice of Fig. 1(a). Specifically, we consider the possibility that when creating one effective site from the two molecules in a dimer, the charge density on the two ET molecules within a dimer are not equal, i.e. there exists a $\mathrm{CO}$ state similar to that found in $\theta$-(ET) [7]. We show below explicit calculations where the presence of $\mathrm{CO}$ does indeed increase the strength of the AFM.

\section{Results and Discussion}

\subsection{Anisotropic triangular half-filled band}

We first present results for the half-filled effective model of Fig. 1(b). The Hamiltonian is

$$
\begin{aligned}
H & =-t_{1} \sum_{\langle i j\rangle, \sigma}\left(c_{j, \sigma}^{\dagger} c_{i, \sigma}+c_{i, \sigma}^{\dagger} c_{j, \sigma}\right) \\
& -t_{2} \sum_{[i j], \sigma}\left(c_{j, \sigma}^{\dagger} c_{i, \sigma}+c_{i, \sigma}^{\dagger} c_{j, \sigma}+U \sum_{i} n_{i, \uparrow} n_{i, \downarrow},\right.
\end{aligned}
$$

where $\langle i j\rangle([i j])$ indicate nearest-neighbor bonds along the solid (dotted) lines of Fig. 1(b). The results shown were obtained for the ground state using the Constrained Path Monte Carlo (CPMC) method [11]. The CPMC method was checked against exact results for a small cluster $(\mathrm{N}=12$ sites) and found to be quite accurate. To quantify the strength of the AFM, we calculate the static spin structure factor, $S(q) . S(q)$ is peaked at $q=$ $(\pi, \pi)$ in the AFM state. The sublattice magnetization $M$ may then be defined as $M^{2}=3 S(\pi, \pi) / N$. In the square-lattice Heisenberg model $(U \rightarrow \infty)$, $M \approx 0.308$ [12]. In Fig. 2 we plot $M$ as a function of $t_{2} / t_{1}$. Morita et al found a $M$ of $\sim 0.1$ at $t_{2} / t_{1}=$ 
0.5 and $U=6$, and a transition to a nonmagnetic state at $t_{2} / t_{1} \sim 0.6[10]$. Note that for these values of $t_{2} / t_{1}$, Hartree-Fock erroneously predicts nearly saturated AFM moments [3]. While we have not yet performed detailed finite-size scaling of the CPMC calculations, our present results are consistent with reference [10], as the points plotted in Fig. 2 are upper bounds to the scaled values of $M$. $\kappa$ - $(\mathrm{ET})_{2} \mathrm{Cl}$ with $t_{2} / t_{1} \sim 0.73[4]$ clearly should be nonmagnetic within this model.

\subsection{Quarter-filled model: $C O$ and $A F M$}

We now return to the quarter-filled lattice structure shown in Fig. 1(a), within the following extended Hubbard Hamiltonian:

$$
\begin{aligned}
H & =-\sum_{\langle i j\rangle, \sigma} t_{i j} c_{i, \sigma}^{\dagger} c_{j, \sigma}+U \sum_{i} n_{i, \uparrow} n_{i, \downarrow} \\
& +V \sum_{\langle i j\rangle} n_{i} n_{j}+\sum_{i, \sigma} \epsilon_{i, \sigma} n_{i, \sigma}
\end{aligned}
$$

We compare the spin-spin correlations of Eq. 1, with and without the presence of CO. Our goal is to establish that long range commensurate AFM can indeed occur in the $\kappa$-lattice in the presence of CO.

We first show exact diagonalization data for spin-spin correlations on the small quarter-filled lattice (16 sites and 8 holes) shown in Fig. 3. This lattice is equivalent to that of Fig. 1(a) with dimer bonds indicated by thick lines. Dimer 1 in Fig. 3 is connected to dimer 2 and 4 via effective $t_{1}$ bonds, and dimer 6 via effective $t_{2}$ bonds. Superimposed on the lattice of Fig. 3 is a horizontal stripe CO of the type found in $\theta$-(ET), adapted for the $\kappa$ (ET) lattice. This CO state is parametrized by the site energy terms $\epsilon_{i, \sigma}$ in Eq. 2. Note that in the actual systems $\mathrm{CO}$ is driven by $V$ as well as electron-molecular vibration coupling, and the site energies in Eq. 2 merely model this mechanism. The coupled bond-charge-spin nature of the $\mathrm{CO}$ can then in principle lead to effective exchange integrals between the dimers that are very different from those for the uniform system without CO. From charge considerations alone, once the $\mathrm{CO}$ insulating state is reached from the metallic state, there occur three different kinds of nearest neighbor intermolecular bonds, which we label as 1-1, $1-0$ and $0-0$, where the numbers 0 and 1 denote populations $0.5+\delta$ and $0.5-\delta$, respectively. The bond orders $\left\langle c_{i, \sigma}^{\dagger} c_{j, \sigma}+\right.$ h.c. $\rangle$ corresponding to the different kinds of bonds are different, and consequently the effective interdimer exchange integrals between the dimers of Fig. $1(\mathrm{a})$ in $\kappa-\mathrm{ET}_{2} \mathrm{X}$ with $\mathrm{CO}$ are also different. The effective Heisenberg $J$ between dimer 1 and dimer 2 is increased due to

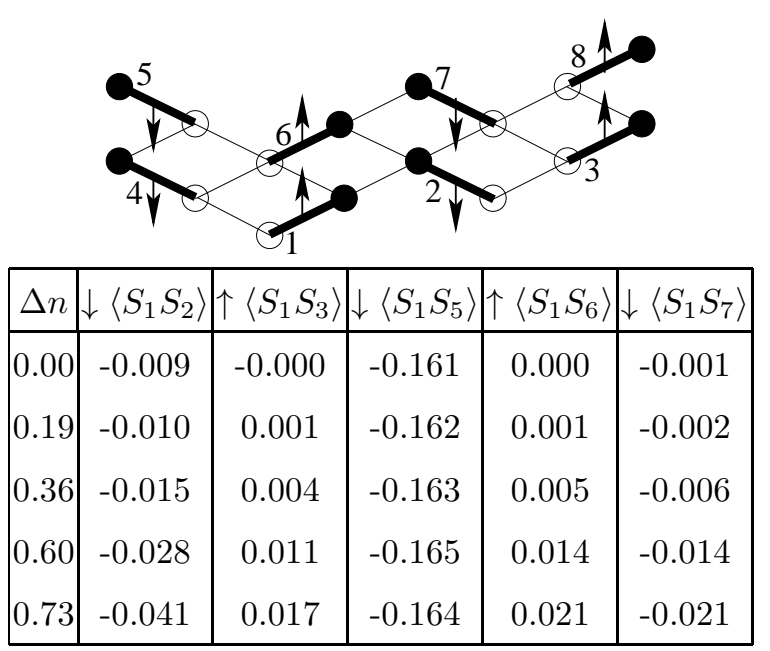

Fig. 3. Horizontal stripe $\mathrm{CO}$ within the $\kappa$-(ET) lattice, showing the spin ordering found in a 16-site exact calculation. Bonds drawn with thick lines are dimer bonds; $t_{q}$ bonds are included in the calculation, but not drawn for clarity. The charge densities of the sites denoted by the unfilled circles are smaller than the ones denoted by the black circles in the CO state. Spin-spin correlations between non-equivalent pairs of dimers are shown in the table, as a function of the CO strength, $\Delta n=n_{\text {big }}-n_{\text {small }}$, where $n_{\text {big }}$ and $n_{\text {small }}$ are the large and small charge densities, respectively. Hamiltonian parameters are as in Fig. 1 (a), with $U=0.7 \mathrm{eV}$ and $V=0.2 \mathrm{eV}$. AFM correlations are strengthened in the $\mathrm{CO}$ state.

the stronger 1-1 bond, while the coupling between dimer 1 and dimer 4 is weakened, reducing the amount of frustration present, and strengthening the AFM ordering indicated by arrows.

The table in Fig. 3 shows the exact spin-spin correlations, comparing uniform $(\epsilon=0$ in Eq. 2) and CO states. To enable comparison with the halffilled band, the correlations shown are spin-spin correlations between dimers, i.e. for dimers with sites $(1,2)$ and $(3,4)$, the dimer-dimer correlation is simply $\left\langle S_{1} S 3\right\rangle+\left\langle S_{1} S_{4}\right\rangle+\left\langle S_{2} S_{3}\right\rangle+\left\langle S_{2} S_{4}\right\rangle$. Once $\mathrm{CO}$ is present, the spin-spin correlations clearly reflect the AFM state indicated by the arrows, and increasing the CO strength continuously strengthens the AFM. Note that the molecular sites with small charge density ("0" above) do have spin density, which is aligned ferromagnetically with the other site in the dimer. The weakness of antiferromagnetic correlations for the uniform lattice with no $\mathrm{CO}$ is obvious, in agreement with the $1 / 2$-filled calculation of Fig. 2. In the CO state, spin-spin correlations corresponding to the 1-0 intradimer bonds are necessarily ferromagnetic now (the same electron occupies both sites), but so are the spinspin correlations corresponding to interdimer 10 bonds, thereby minimizing the spin frustration that characterizes the effective lattice of Fig. 1(b). Because of the large finite size effects associated 

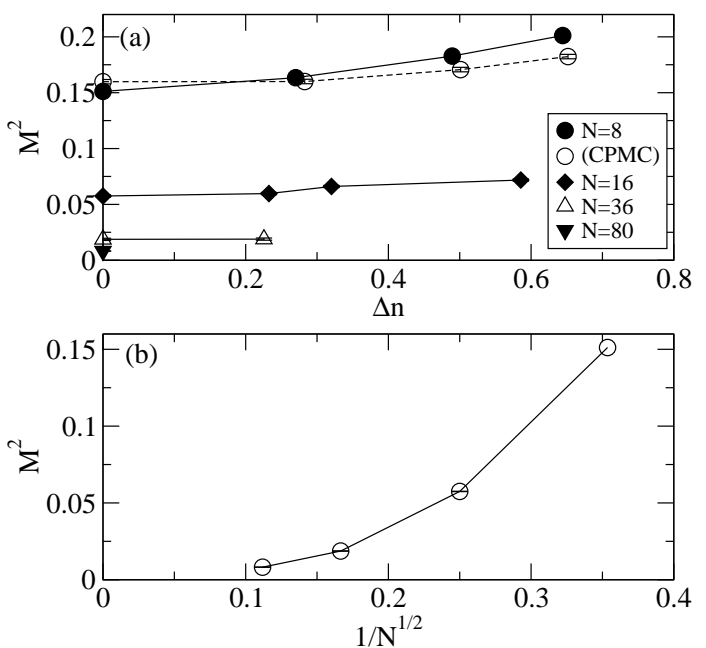

Fig. 4. (a) Sublattice magnetization for quarter-filled lattices of Fig. 1(a), with $U=0.7 \mathrm{eV}$ and $V=0 \mathrm{eV}$. Horizontal axis indicates the amount of $\mathrm{CO}$ as in Fig. 3. Filled (open) circles indicate exact (CPMC) calculation for $N=8$ dimers. (b) Size dependence of $M^{2}$ for the quarter-filled lattice with no $\mathrm{CO}$. The large-lattice limit of $M$ vanishes.

with our very small lattice, we now turn to quantum Monte Carlo simulations of larger clusters.

\subsection{Quarter-filled model: large-lattice results}

We have performed direct calculations on larger quarter-filled lattices (up to 160 lattice sites/80 dimers), again using the CPMC method. For increased accuracy with the CPMC method, we have performed these calculations for $V=0$. Results for these calculations are shown in Fig. 4. Fig. 4(a) shows $M$ vs. $\Delta n$ for several lattice sizes (including a comparison with exact results for the 8 dimer lattice of Fig. 3). As in Fig. 3, we find that $\mathrm{CO}$ strengthens the AFM slightly. The $N=8$ results also indicate that CPMC may underestimate the strengthening of the AFM with charge order, in contrast to mean field calculations which overestimate the AFM.

Fig. 4(b) shows the finite-size scaling of $M$ for the uniform (no CO) quarter-filled lattice. We find that the scaled value of $M$ is essentially zero, consistent with Fig. 2 and the results of Morita et al. $[10]$.

\section{Conclusions}

In conclusion, we have investigated the AFM state in the triangular lattice appropriate for $\kappa$ (ET). We find two principle results: First, within the best estimate of model parameters appropriate for $\kappa$-(ET), the staggered magnetic moment ob- tained in either the quarter-filled or half-filled effective models is much weaker than experimental values, and as frustration $t_{2} / t_{1}$ increases from zero, the magnetization drops rapidly. Second, we have presented calculations assuming the presence of $\mathrm{CO}$, and find that the magnetization increases continuously as the $\mathrm{CO}$ is strengthened. The strengthening of the AFM state occurs due to reduced frustration in the presence of $\mathrm{CO}$. Our result that strong AFM is absent in the $\kappa$-lattice is in agreement with earlier work. One intriguing question that arises now is that experiments to date have not indicated $\mathrm{CO}$ in $\kappa$ salts. This could be because of our ignoring electron-lattice interactions in the Hamiltonian (2). As shown elsewhere, such interactions cause strong modulations of the hopping integrals in the presence of $\mathrm{CO}$ in the $1 / 4$-filled lattices $[6,7]$. It is possible that in the real materials, the differences in $\mathrm{CO}$ are too small for easy detection, but the resultant hopping integral modulations are large and reduce frustration. We are currently pursuing self-consistent calculations of hopping integrals within model Hamiltonians that incorporate electron-lattice interactions.

\section{Acknowledgments}

Work in Arizona was supported by NSF DMR0406604. RTC acknowledges support from the ERC Center for Computational Sciences at MSU.

\section{References}

[1] R. H. McKenzie, Comments Cond. Matt. Phys. 18 (1998) 309.

[2] J. Schmalian, Phys. Rev. Lett. 81 (1998) 4232.

[3] H. Kino, H. Fukuyama, J. Phys. Soc. Jpn. 65 (1996) 2158.

[4] T. Mori, H. Mori, S. Tanaka, Bull. Chem. Soc. Jpn. 72 (1999) 179.

[5] K. Miyagawa, K. Kanoda, A. Kawamoto, Chem. Rev. 104 (2004) 5635.

[6] R. T. Clay, S. Mazumdar, D. K. Campbell, Phys. Rev. B 67 (2003) 115121.

[7] R. T. Clay, S. Mazumdar, D. K. Campbell, J. Phys. Soc. Jpn. 71 (2002) 1816.

[8] J. Merino, R. H. McKenzie, J. B. Marston, C. H. Chung, J. Phys.:Condens. Matter 11 (1999) 2965.

[9] A. E. Trumper, Phys. Rev. B 60 (1999) 2987.

[10] H. Morita, S. Watanabe, M. Imada, J. Phys. Soc. Jpn. 71 (2002) 2109.

[11] S. Zhang, J. Carlson, J. E. Gubernatis, Phys. Rev. B 55 (1997) 7464 .

[12] A. W. Sandvik, Phys. Rev. B 56 (1997) 11678. 